Supporting Information for:

\title{
Aryl Migration on Graphene
}

Maggie He and Timothy M. Swager*

Department of Chemistry, Massachusetts Institute of Technology, 77 Massachusetts Avenue, Cambridge, MA 02139, USA

\section{Table of Contents}

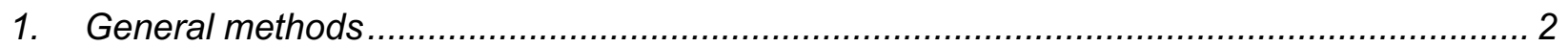

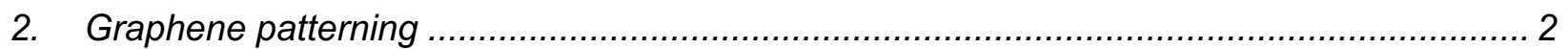

3. Functionalization of graphene with iodonium salts ................................................... 2

4. Characteristic Raman bands of unfunctionalized and covalently functionalized graphene... 3

5. XPS of unfunctionalized and 4-trifluoromethylphenyl functionalized graphene................... 4

6. FTIR spectra of functionalized graphene ................................................................... 5

7. Raman analysis of 4-trifluoromenthylphenyl functionalized graphene .............................. 6

8. Raman analysis of phenyl functionalized graphene .................................................... 7

9. Raman analysis of 4-methoxyphenyl functionalized graphene ...................................... 8

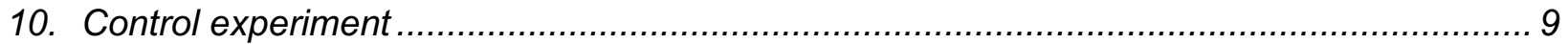

11. Raman analysis of 4-methoxyphenyl functionalized graphene before and after migration ... 9

12. Stability of methoxyphenyl group in ammonium persulfate solution................................. 10

13. XPS analysis of 4-methoxyphenyl functionalized graphene........................................ 11

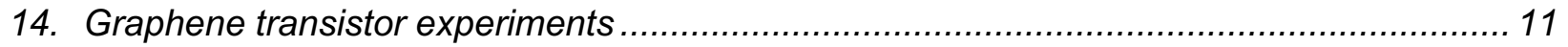

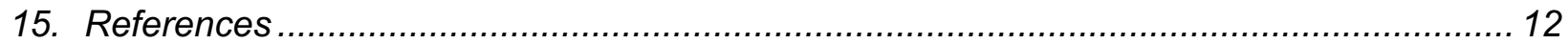




\section{General methods}

All reactions were carried out in oven dried glassware. Anhydrous, inhibitor-free THF was dried over $4 \AA$ molecular sieves. Bis(4-trifluoromethylphenyl)iodonium tetrafluoroborate, diphenyliodonium triflate and bis(4-methoxyphenyl)iodonium tetrafluoroborate were synthesized according to literature procedure. ${ }^{1}$ Monolayer graphene on $\mathrm{SiO}_{2}(300 \mathrm{~nm}) / \mathrm{Si}$ wafer $(10 \mathrm{~mm} \times 10$ $\mathrm{mm}$ ) and on copper was purchased from Graphene Square. Raman spectra were measured with a Horiba Jobin-Yvon LabRam (Model HR 800) Raman confocal microscope with a $532 \mathrm{~nm}$ laser excitation and an automated stage. The diffraction limited spot size was $0.36 \mu \mathrm{m}$ and laser power was $0.9 \mathrm{~mW}$. Raman maps were recorded with the same parameter on $30 \mu \mathrm{m} \times 30 \mu \mathrm{m}$ area with a $1 \mu \mathrm{m}$ step size. These maps were processed with the LabSpec 6 software. The sample position and coordinates where Raman spectra and maps were taken were recorded and used to ensure successive measurements were made around the same location. Because the graphene samples need to be removed from the spectrometer stage to perform chemical processes, the location of subsequent analysis may be slightly off (usually $<1 \mu \mathrm{m}$ ). Attenuated total reflectance-Fourier transform infrared (ATR-FTIR) spectra were acquired using Thermo Scientific Nicolet 6700 FTIR with a Ge crystal. X-ray photoelectron spectroscopy (XPS) measurements were performed on a Physical Electronics Versaprobe II X-ray photoelectron spectrometer with a hemispherical

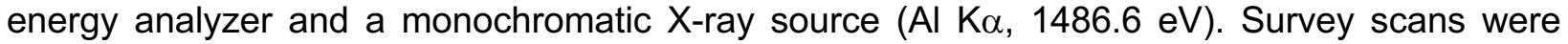
collected with a X-ray setting of $200 \mu \mathrm{m}, 50 \mathrm{~W}$, and $15 \mathrm{kV}$ and a pass energy of $187.85 \mathrm{eV}$ at a base pressure of $10^{-9}$ Torr. XPS spectra were processed using the CasaXPS software.

\section{Graphene patterning}

Copper pattern on monolayer graphene was prepared by evaporation of $50 \mathrm{~nm}$ of copper (Kurt J. Lesker, 99.999\%) at a deposition rate of $2 \AA / s$ at $10^{-6}$ Torr using an electron-beam evaporator (AJA International Inc., model: AJA ATC) in a class 10,000 clean room. A copper grid (Ultra Fine Circular Aperture 2000 Mesh, Ted Pella, INC. pitch $12.5 \mu \mathrm{m}$, hole width $6.5 \mu \mathrm{m}$, bar width $6 \mu \mathrm{m}$ ) was used as the shadow mask for patterning. Graphene samples patterned with copper were used immediately after copper deposition. After arene functionalization with iodonium salts, functionalization was confirmed by Raman analysis. Copper islands on monolayer graphene was removed by etching with $0.10 \mathrm{M}$ ammonium persulfate solution for $2 \mathrm{~min}$, cleaned thoroughly with water, and dried under a stream of $\mathrm{N}_{2}$.

\section{Functionalization of graphene with iodonium salts}

Functionalization of monolayer graphene was carried out in a nitrogen filled glovebox with an oxygen level below $1 \mathrm{ppm}$. Graphene was submerged in a solution of sodium naphthalide $(0.031$ $\mathrm{M}$ ) in degassed, anhydrous THF for $1 \mathrm{~min}$ at room temperature. The reductant solution was removed using a glass pipet and the reduced graphene was immersed in a solution of iodonium salt $(0.010 \mathrm{M})$ in THF for $1 \mathrm{~min}$ at room temperature. The graphene was removed from the glovebox, thoroughly cleaned with $\mathrm{EtOH}: \mathrm{H}_{2} \mathrm{O}(10: 1)$ and dried under a stream of $\mathrm{N}_{2}$. Copperpatterned graphene as well as CVD graphene on copper were functionalized similarly. 
4. Characteristic Raman bands of unfunctionalized and covalently functionalized graphene
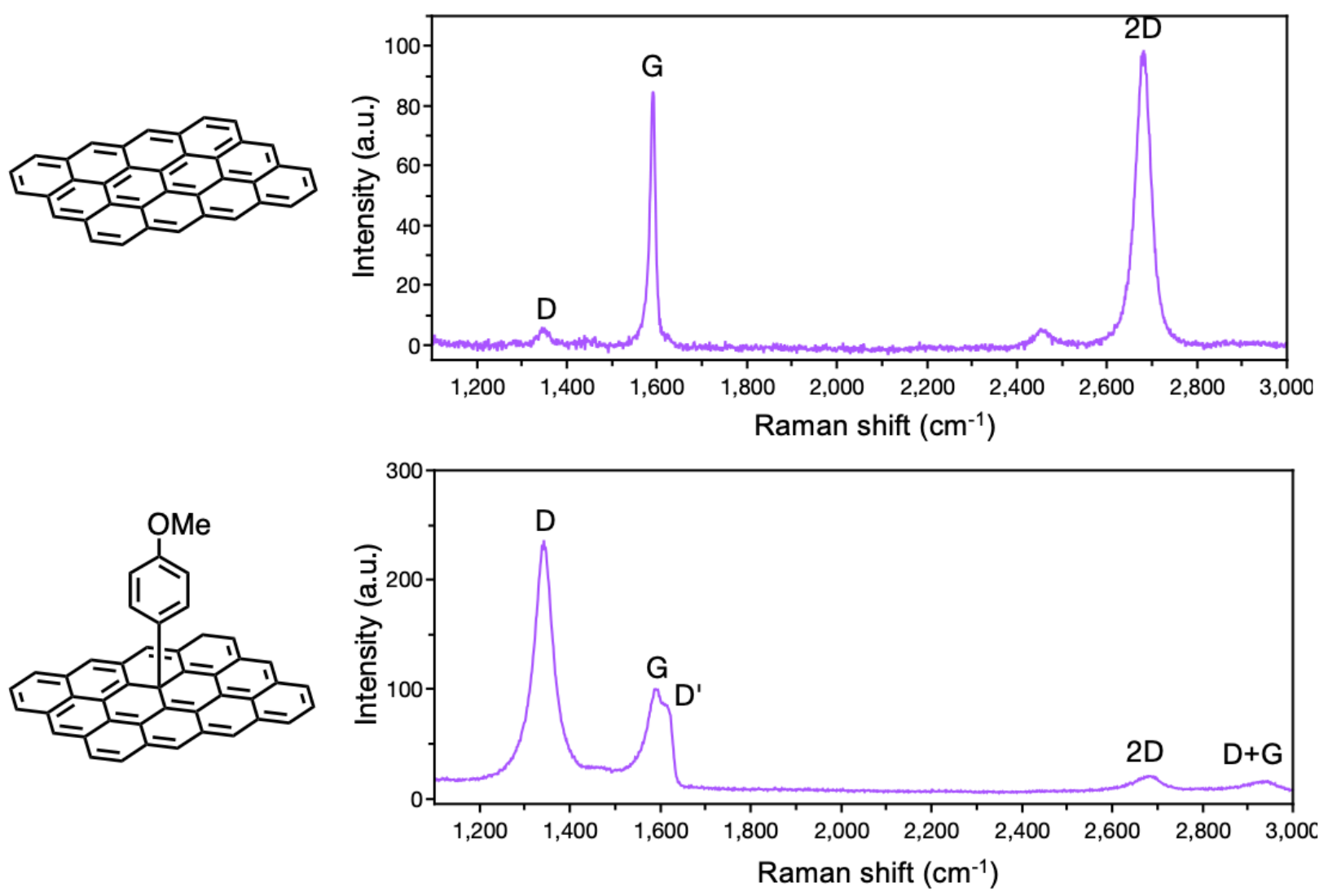

Figure S1. Raman spectra of unfunctionalized and covalently functionalized graphene. 
5. XPS of unfunctionalized and 4-trifluoromethylphenyl functionalized graphene
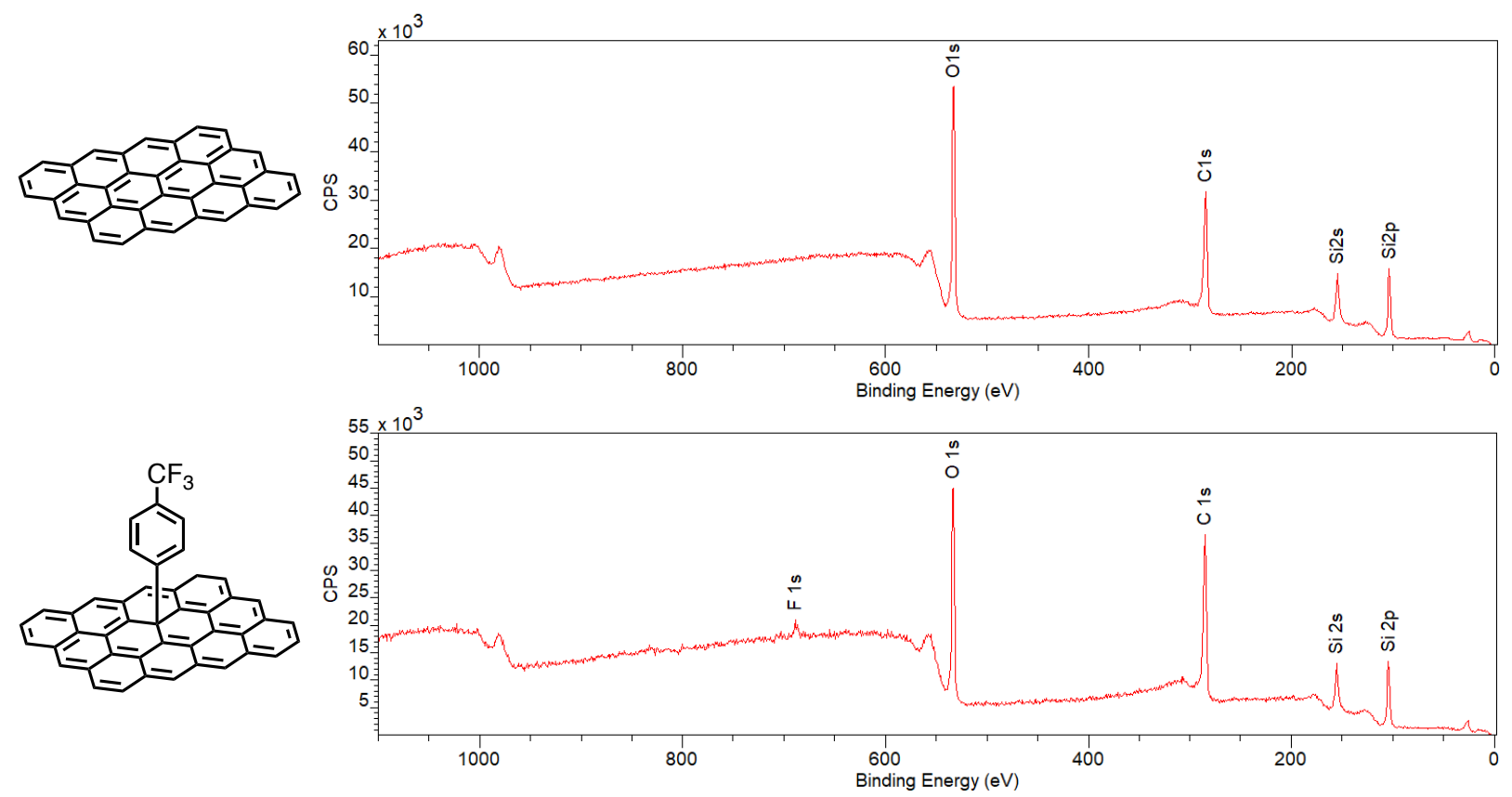

Figure S2. XPS survey spectra of unfunctionalized (top) and 4-trifluoromethylphenyl functionalized graphene (bottom).

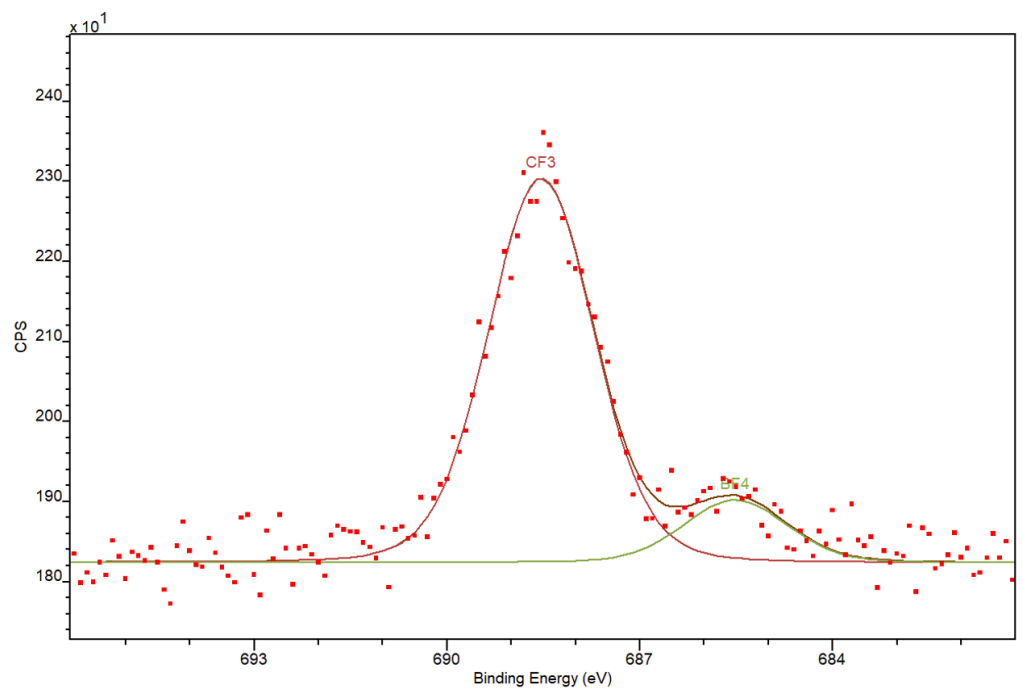

Figure S3. Peak fitting of the $F$ 1s high resolution spectra of 4-trifluoromethylphenyl functionalized graphene. The peak at $688.7 \mathrm{eV}$ belongs to the trifluomethyl group. The small peak at $685.5 \mathrm{eV}$ arises from residual $\mathrm{BF}_{4}{ }^{-}$salt. 


\section{FTIR spectra of functionalized graphene}

The characterization of functional groups on graphene by FTIR was complicated by the large background signals obscuring the weak characteristic IR peaks from the underlaying $\mathrm{SiO}_{2}$ layer on the silicon wafer. The $\mathrm{CF}_{3}$ stretch at $1337 \mathrm{~cm}^{-1}$ in $\mathrm{SLG}^{-P h C F_{3}}$ and $\mathrm{C}-\mathrm{O}$ stretch at $1261 \mathrm{~cm}^{-1}$ in SLG-PhOMe were observed in the IR spectra when graphene is deposited on copper, which provided a much lower spectral background.

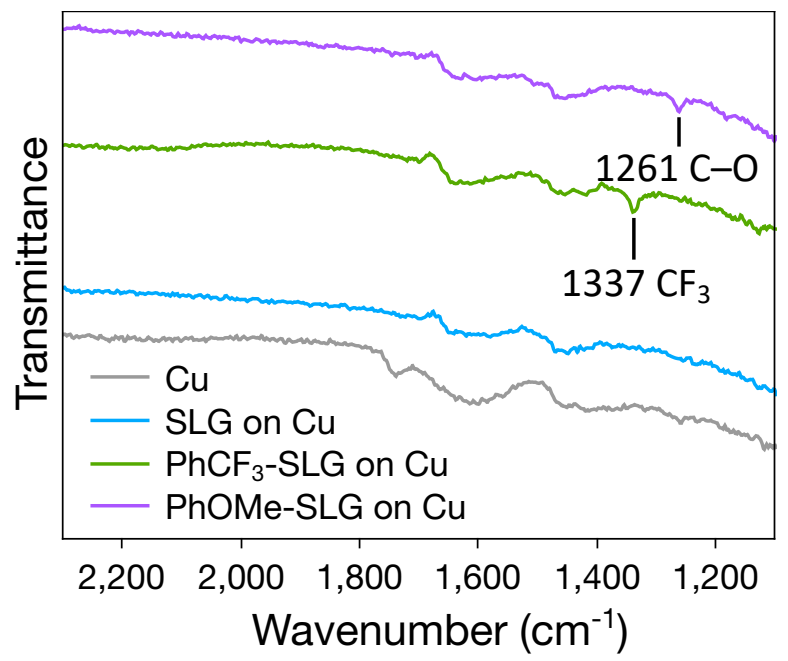

Figure S4. ATR-FTIR of unfunctionalized and functionalized graphene on copper. 


\section{Raman analysis of 4-trifluoromenthylphenyl functionalized graphene}
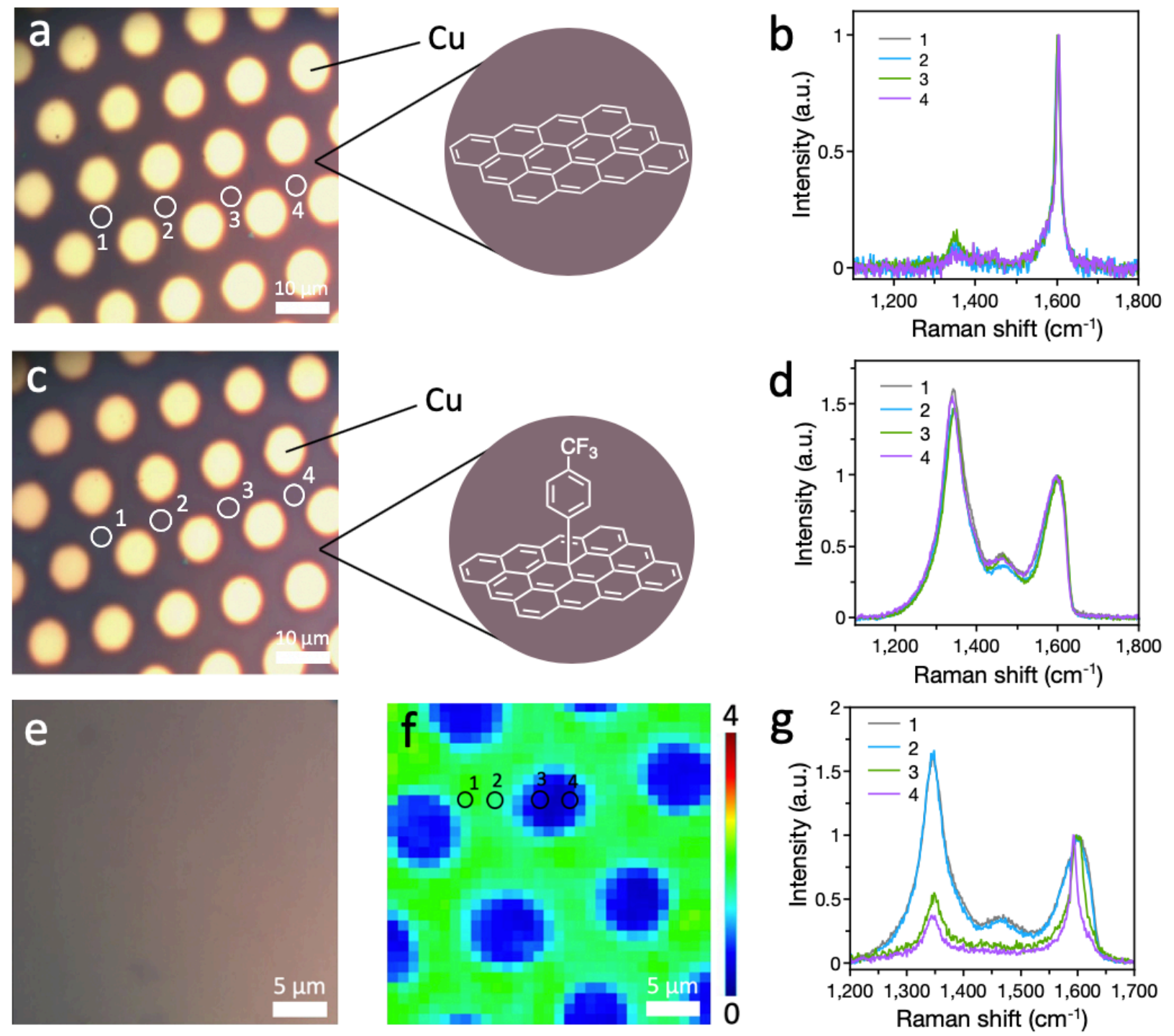

Figure S5. (a) Optical image of unfunctionalized graphene patterned with $\mathrm{Cu}$ (bright yellow circle, diameter $6.5 \mu \mathrm{m}$ ). (b) Raman spectra of the labeled areas in a. (c) Optical image of copper patterned graphene functionalized with 4-trifluoromethylphenyl groups. (d) Raman spectra of the labeled areas in c. (e) Optical image of functionalized graphene after $\mathrm{Cu}$ etch with $0.1 \mathrm{M}$ ammonium persulfate solution. (f) Raman map of $I_{D} / l_{G}$ of a $30 \mu \mathrm{m} \times 30 \mu \mathrm{m}$ area. The patterned functionalized and unfunctionalized regions are clearly distinguishable (unfunctionalized regions are in blue and functionalized regions are in green). (g) Spectra retrieved from the labeled areas in Raman map f. 


\section{Raman analysis of phenyl functionalized graphene}
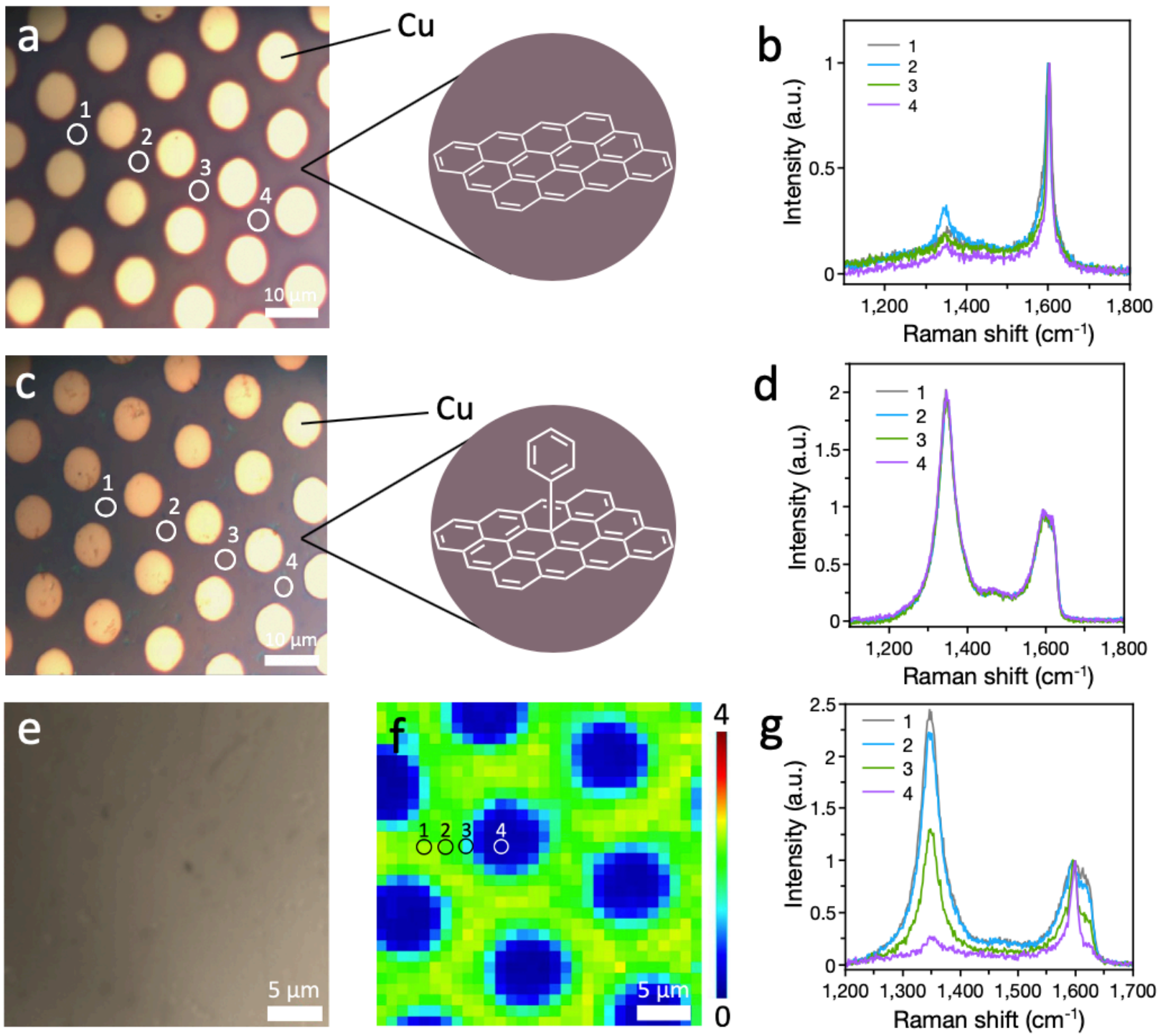

Figure S6. (a) Optical image of unfunctionalized graphene patterned with $\mathrm{Cu}$ (bright yellow circle, diameter $6.5 \mu \mathrm{m}$ ). (b) Raman spectra of the labeled areas in a. (c) Optical image of copper patterned graphene functionalized with phenyl groups. (d) Raman spectra of the labeled areas in c. (e) Optical image of functionalized graphene after $\mathrm{Cu}$ etch with $0.1 \mathrm{M}$ ammonium persulfate solution. (f) Raman map of $I_{D} / I_{G}$ of a $30 \mu \mathrm{m} \times 30 \mu \mathrm{m}$ area. The patterned functionalized and unfunctionalized regions are clearly distinguishable (unfunctionalized regions are in blue and functionalized regions are in green). (g) Spectra retrieved from the labeled areas in Raman map f. 


\section{Raman analysis of 4-methoxyphenyl functionalized graphene}
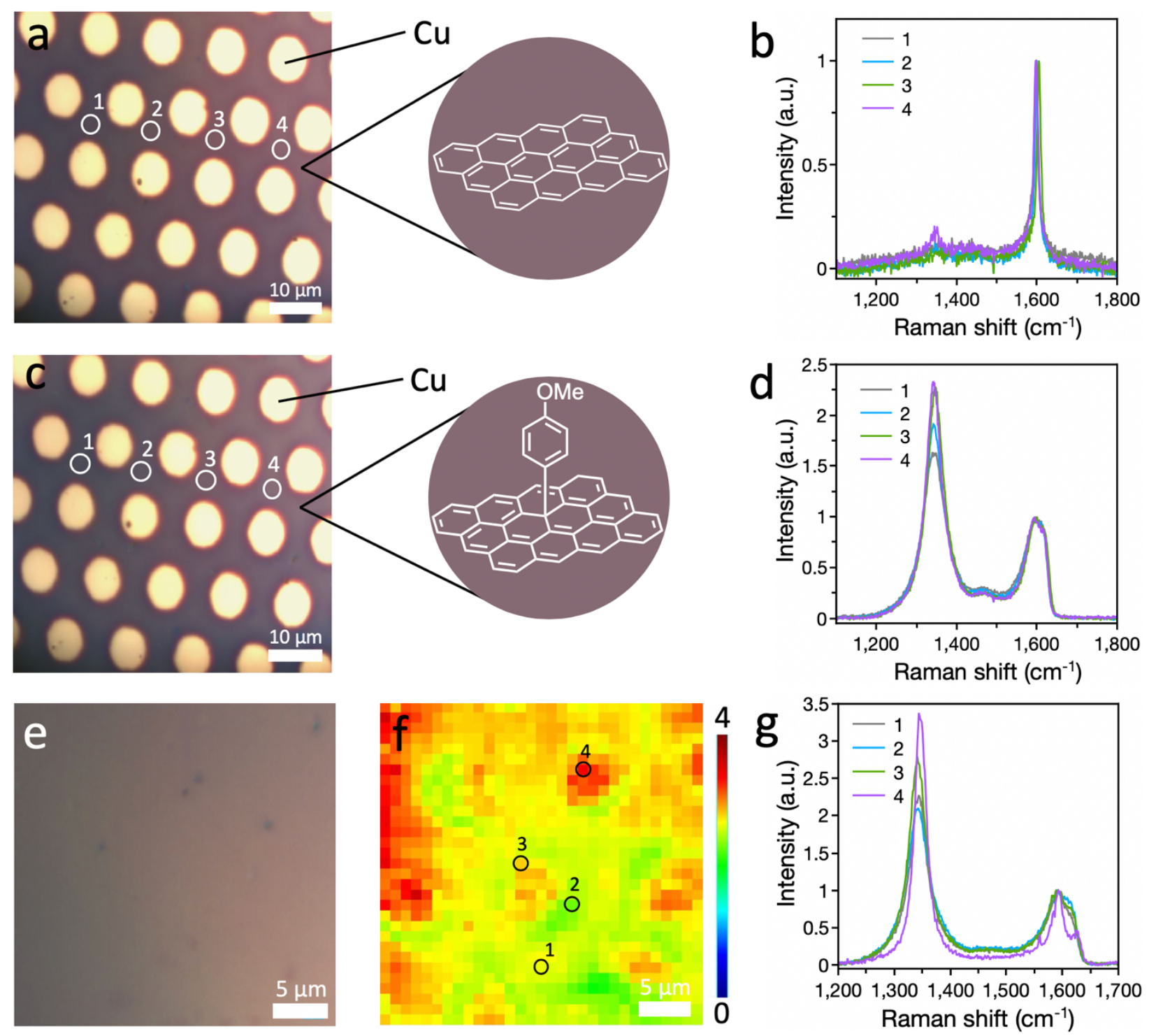

Figure S7. (a) Optical image of unfunctionalized graphene patterned with $\mathrm{Cu}$ (bright yellow circle, diameter $6.5 \mu \mathrm{m}$ ). (b) Raman spectra of the labeled areas in a. (c) Optical image of copper patterned graphene functionalized with 4-methoxyphenyl groups. (d) Raman spectra of the labeled areas in c. (e) Optical image of functionalized graphene after $\mathrm{Cu}$ etch with $0.1 \mathrm{M}$ ammonium persulfate solution. (f) Raman map of $I_{D} / I_{G}$ of a $30 \mu \mathrm{m} \times 30 \mu \mathrm{m}$ area. The 4methoxyphenyl groups have migrated into the unfunctionalized region and the patterned functionalized and unfunctionalized regions are scrambled. (g) Spectra retrieved from the labeled areas in Raman map f. 


\section{Control experiment}

To confirm that the etchant used to etch copper was not damaging the graphene and causing the observed increase in $I_{D} / I_{G}$ across the graphene. We carried out a control experiment where we subjected the graphene patterned with copper, with no additional functionalization, to $0.1 \mathrm{M}$ ammonium persulfate. Raman mapping $\left(I_{D} / I_{G}\right)$ over the etched graphene surface reveals a very similar uniform topography with a small $I_{D} / I_{G}$ comparable that determined for the pristine unfunctionalized graphene.
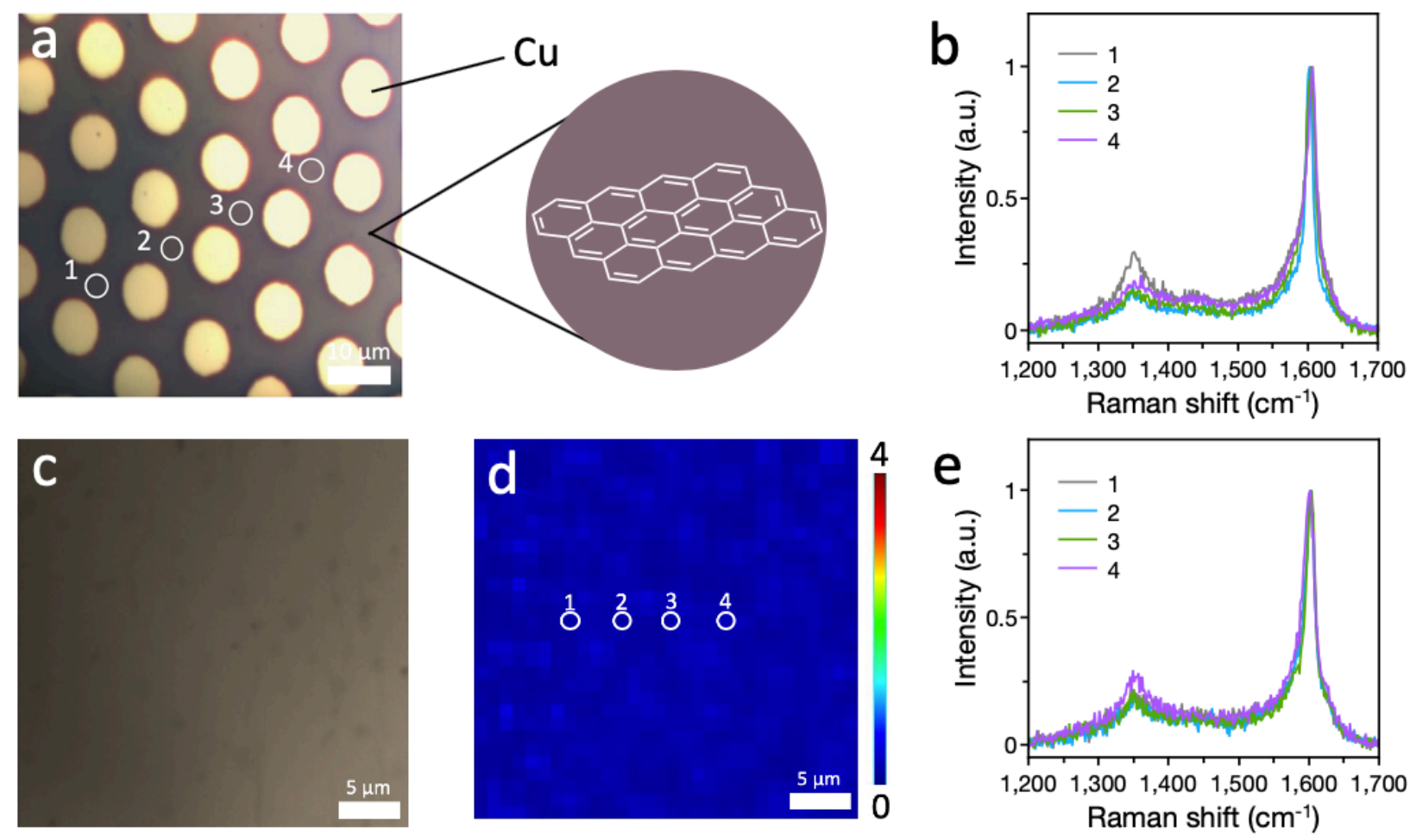

Figure S8. Control experiment showing treatment of graphene with $0.1 \mathrm{M}$ ammonium persulfate solution induce negligible damage to graphene as evident by the comparable intensity of the $D$ band before and after treatment. (a) Optical image of unfunctionalized graphene patterned with $\mathrm{Cu}$ (bright yellow circle, diameter $6.5 \mu \mathrm{m}$ ). (b) Raman spectra of the labeled areas in a. (c) Optical image of unfunctionalized graphene patterned with $\mathrm{Cu}$ after etching with $0.1 \mathrm{M}$ ammonium persulfate solution. (d) Raman map of $I_{D} / I_{G}$ of a $20 \mu \mathrm{m} \times 20 \mu \mathrm{m}$ area. (e) Spectra retrieved from the labeled areas in Raman map d.

\section{Raman analysis of 4-methoxyphenyl functionalized graphene before and after migration}

We analyzed the Raman $I_{D} / I_{G}$ and full width at half maximum (FWHM) of the $D$ band to further support the migration of 4-methoxyphenyl groups from a region of high functionalization to regions with no prior functionalization. Raman studies found that the $\mathrm{I}_{\mathrm{D}} / \mathrm{l}_{\mathrm{G}}$ ratio does not increase linearly as the defects increase in graphene. It follows that the $I_{D} / l_{G}$ increases as the distance between defects decrease, reaching a maximum at a defect distance of approximately $3 \mathrm{~nm}$. Below $3 \mathrm{~nm}$ of defect distance, the $\mathrm{I}_{D} / \mathrm{I}_{\mathrm{G}}$ decreases. Regarding the FWHM of the $\mathrm{D}$ band, it does not change significantly at low defect concentration, but it increases significantly when the defect distance is less than $4 \mathrm{~nm}^{2}$ Comparing four Raman spectra of the functionalized regions before (Figure S7d) 
and after migration (Figure $\mathbf{S 7 g}$ ), we found the average $I_{D} / I_{G}$ ratio of the functionalized region before migration (2.0) was smaller than those after migration (2.6). On the other hand, the FWHM of the Raman spectra showed an average decrease in the FWHM after migration, from 59 to 38. Together, the $I_{D} / I_{G}$ and $F W H M$ of the $D$ band indicate an increase in the defect distance as the 4methoxyphenyl groups are distributed further apart.

\section{Stability of methoxyphenyl group in ammonium persulfate solution}

The stability of methoxyphenyl group in ammonium persulfate was examined through NMR experiments with anisole. We mixed equal volume of $0.1 \mathrm{M}$ anisole in $\mathrm{CDCl}_{3}$ and $0.1 \mathrm{M}$ ammonium persulfate in $\mathrm{D}_{2} \mathrm{O}$ and stirred vigorously in a vial overnight. The $\mathrm{CDCl}_{3}$ layer was carefully taken out and analyzed by ${ }^{1} \mathrm{H}$ NMR. The ${ }^{1} \mathrm{H}$ NMR of the ammonium persulfate treated anisole looks exactly the same as anisole in $\mathrm{CDCl}_{3}$, indicating the stability of methoxyphenyl group in the oxidizing conditions.

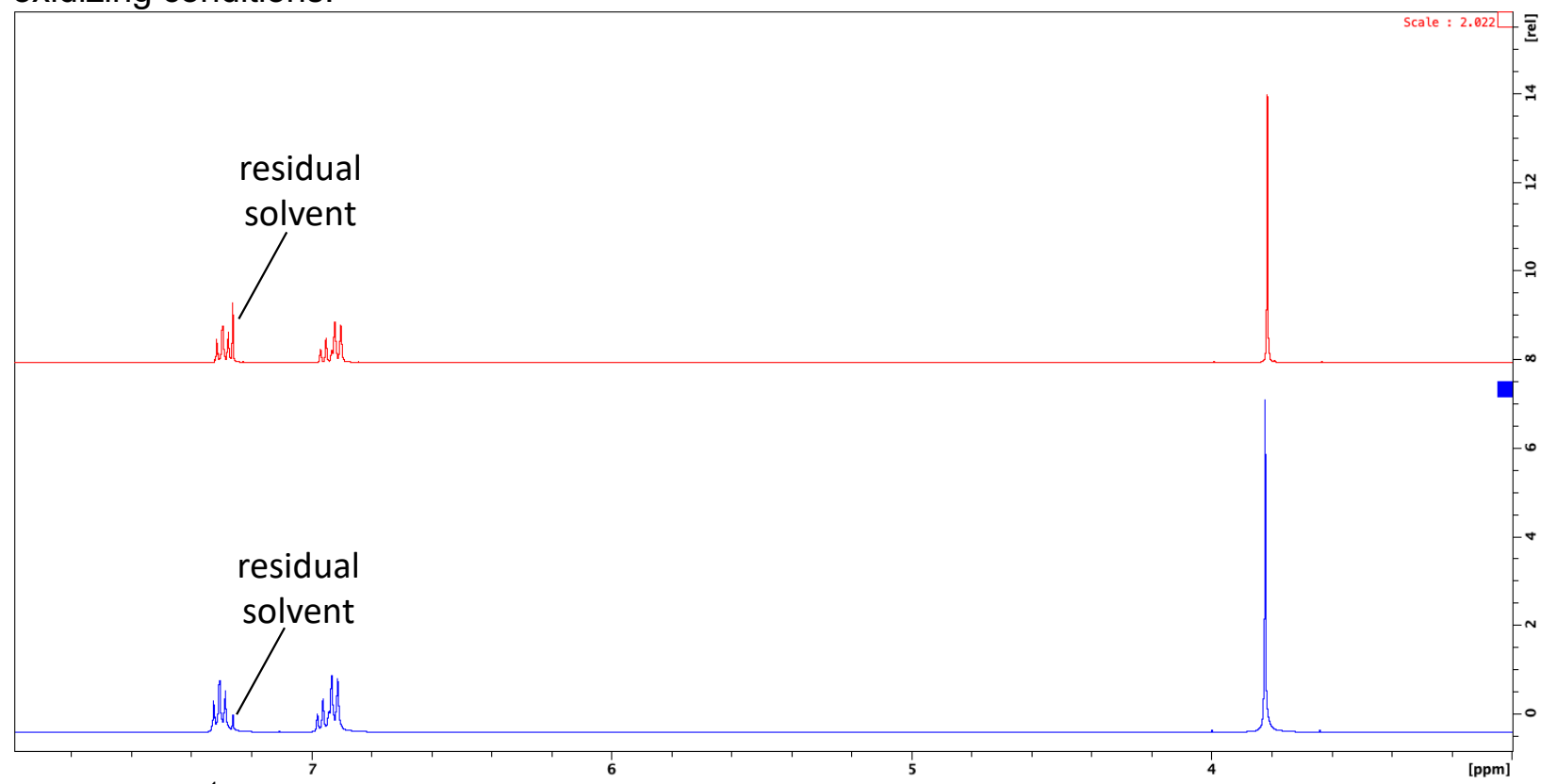

Figure S9. ${ }^{1} \mathrm{H}$ NMR spectra of anisole (lower spectrum) and anisole treated with ammonium persulfate (upper spectrum). 


\section{XPS analysis of 4-methoxyphenyl functionalized graphene}
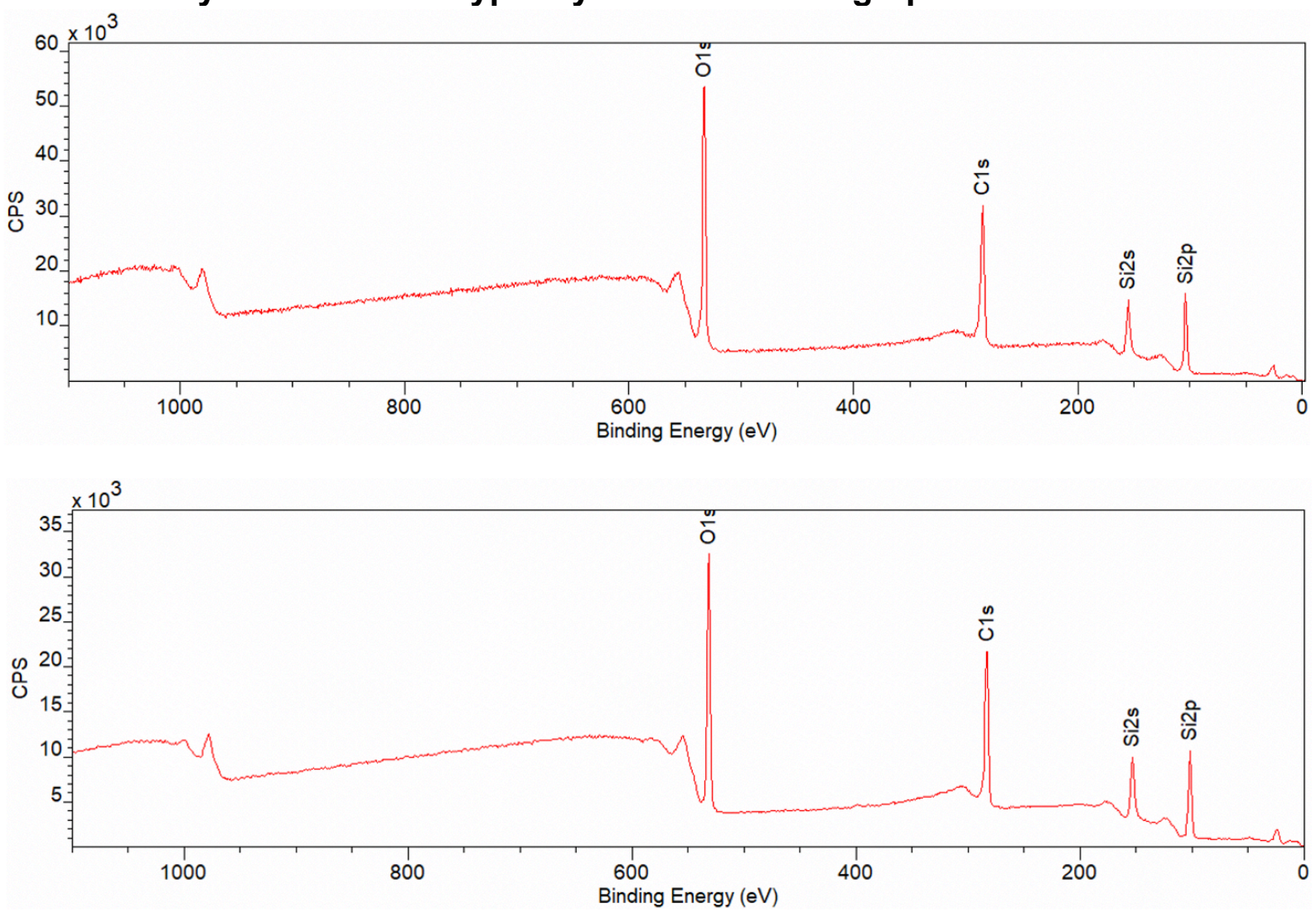

Figure S10. XPS analysis of 4-methoxyphenyl functionalized graphene before (top) and after (bottom) ammonium persulfate treatment. No residual ammonium persulfate was observed on graphene after cleaning with water and thoroughly dried under a stream of $\mathrm{N}_{2}$.

\section{Graphene transistor experiments}

To confirm the doping behavior of graphene, we conducted electrochemically top-gated graphene transistor experiments using a platinum wire top gate and 1-butyl-3-methylimidazolium hexafluorophosphate as the electrolyte. The $\mathrm{I}_{\mathrm{SD}}$ as a function of top-gate voltages $\left(\mathrm{V}_{\mathrm{TG}}\right)$ at 150 $\mathrm{mV}$ bias were plotted in Figure S11. Since the conductivity minimum is displayed to the right of $V_{\mathrm{TG}}=0$, both graphene and 4-methoxyphenyl functionalized graphene are p-doped. 

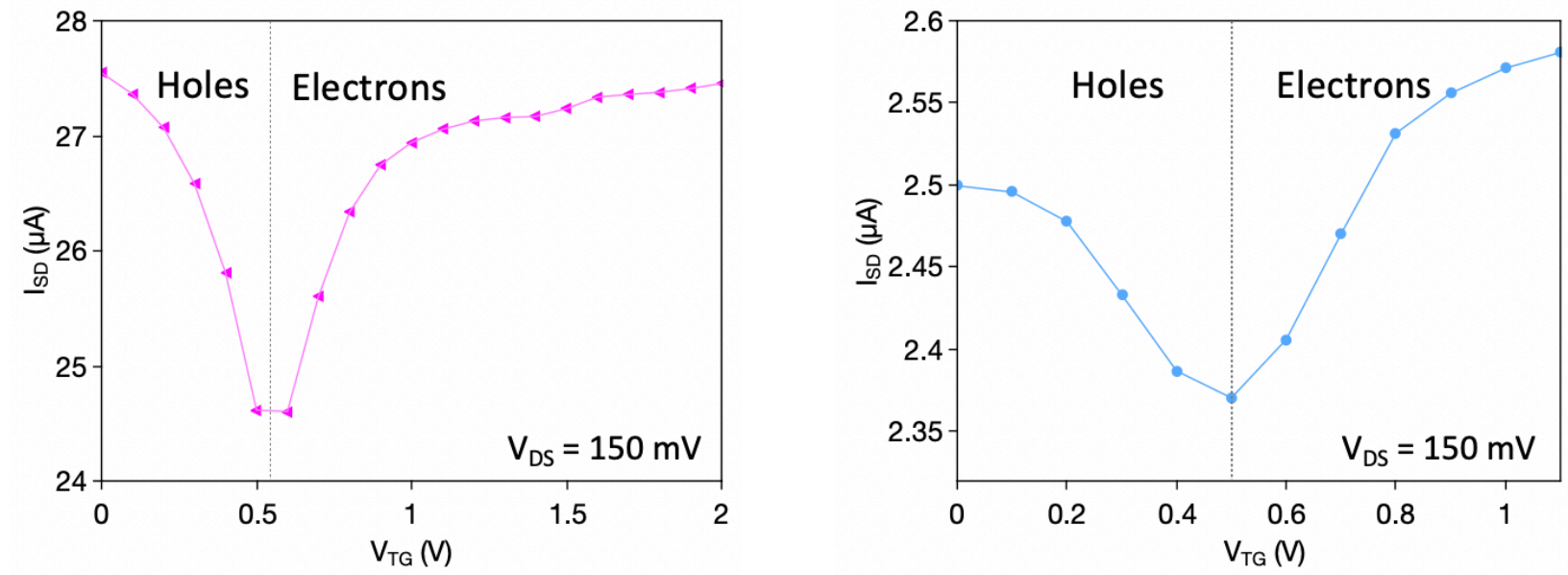

Figure S11. ISD as a function of top-gate voltages $\left(\mathrm{V}_{\mathrm{TG}}\right)$. Left: unfunctionalized graphene. Right: 4-methoxyphenyl functionalized graphene.

\section{References}

(1) He, M.; Swager, T. M. Covalent Functionalization of Carbon Nanomaterials with lodonium Salts. Chem. Mater. 2016, 28 (23), 8542-8549.

(2) Cançado, L. G.; Jorio, A.; Ferreira, E. H. M.; Stavale, F.; Achete, C. A.; Capaz, R. B.; Moutinho, M. V. O.; Lombardo, A.; Kulmala, T. S.; Ferrari, A. C. Quantifying Defects in Graphene via Raman Spectroscopy at Different Excitation Energies. Nano Lett. 2011, 11, 3190-3196. 